\title{
Assessment of changes in lactate measurement with intravascular microdialysis using the trend interchangeability method
}

\author{
Bonnet V. ${ }^{1}$, Gouezel C. ${ }^{1}$, Lorne E. ${ }^{2}$, Gerard J.-L. ${ }^{1}$, Fellahi J.-L. ${ }^{3}$, Fischer M.-O. ${ }^{1}$, French \\ Hemodynamic Team
}

1 University Hospital Caen, Dept of Anaesthesiology \& Intensive Care, Caen, France, 2Amiens University Medical Center, Dept of Anaesthesiology \& Intensive Care, Amiens, France, 3Louis Pradel Academic Hospital, Dept of Anaesthesiology \& Intensive Care, Lyon, France

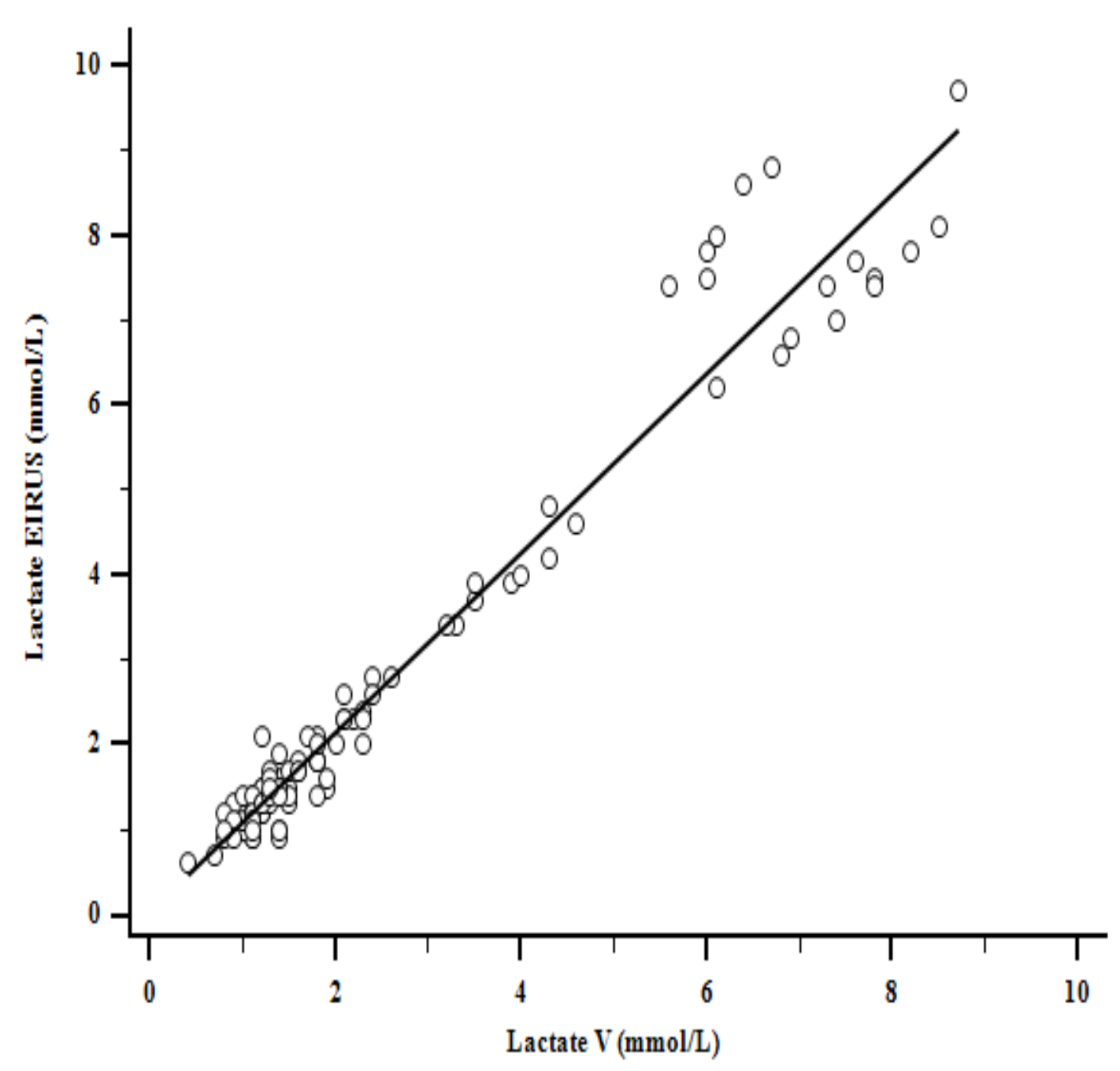

Background and Goal of Study: Blood lactate is a strong predictor of mortality, and it is recommended to monitor its clearance over time during surgery in high-risk patients. We studied the interchangeability of venous lactate measurement through intravenous microdialysis incorporated in a central venous catheter with the reference method on arterial blood sample.

Materials and Methods: Blood lactate measurements from microdialysis and central venous blood were simultaneously recorded in 23 high-risk cardiac surgical patients (Trial Registry Number: NCT02296593). For absolute values, correlation was determined by linear regression, and Bland-Altman for repeated measurements was used to compare bias, precision, and limits of agreement. Changes in lactate measurement were evaluated with four-quadrant plot and trend interchangeability methods.

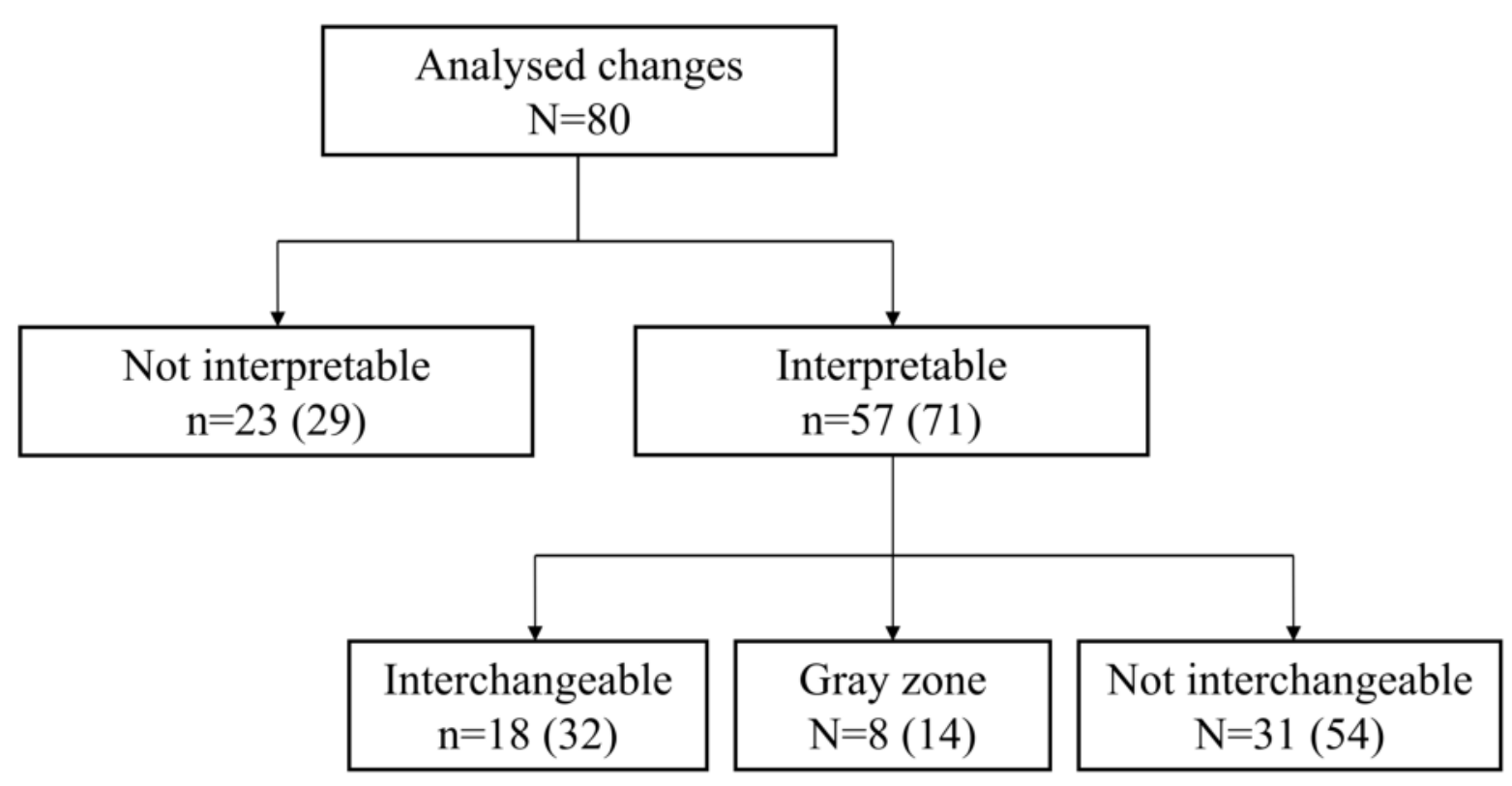

Results and Discussion: For the 22 analysed patients, the central venous catheter was used as usual care without complication. For absolute values $(n=104)$, the correlation coefficient was $0.96(P<0.0001)$. The bias, precision and limits of agreement were $-0.19,0.51$ and -1.20 to $0.82 \mathrm{mmol} \mathrm{l}-1$, respectively. For changes in lactate measurement assessment $(n=80)$, the concordance rate was $94 \%$ with the four-quadrant plot. In contrast, the trend interchangeability method showed that 23 (29) changes of lactate measurements were not interpretable; and among the remaining 57 (71) interpretable changes, 18 (32) were interchangeable, 8 (14) were in the gray zone, and 31 (54) were not interchangeable.

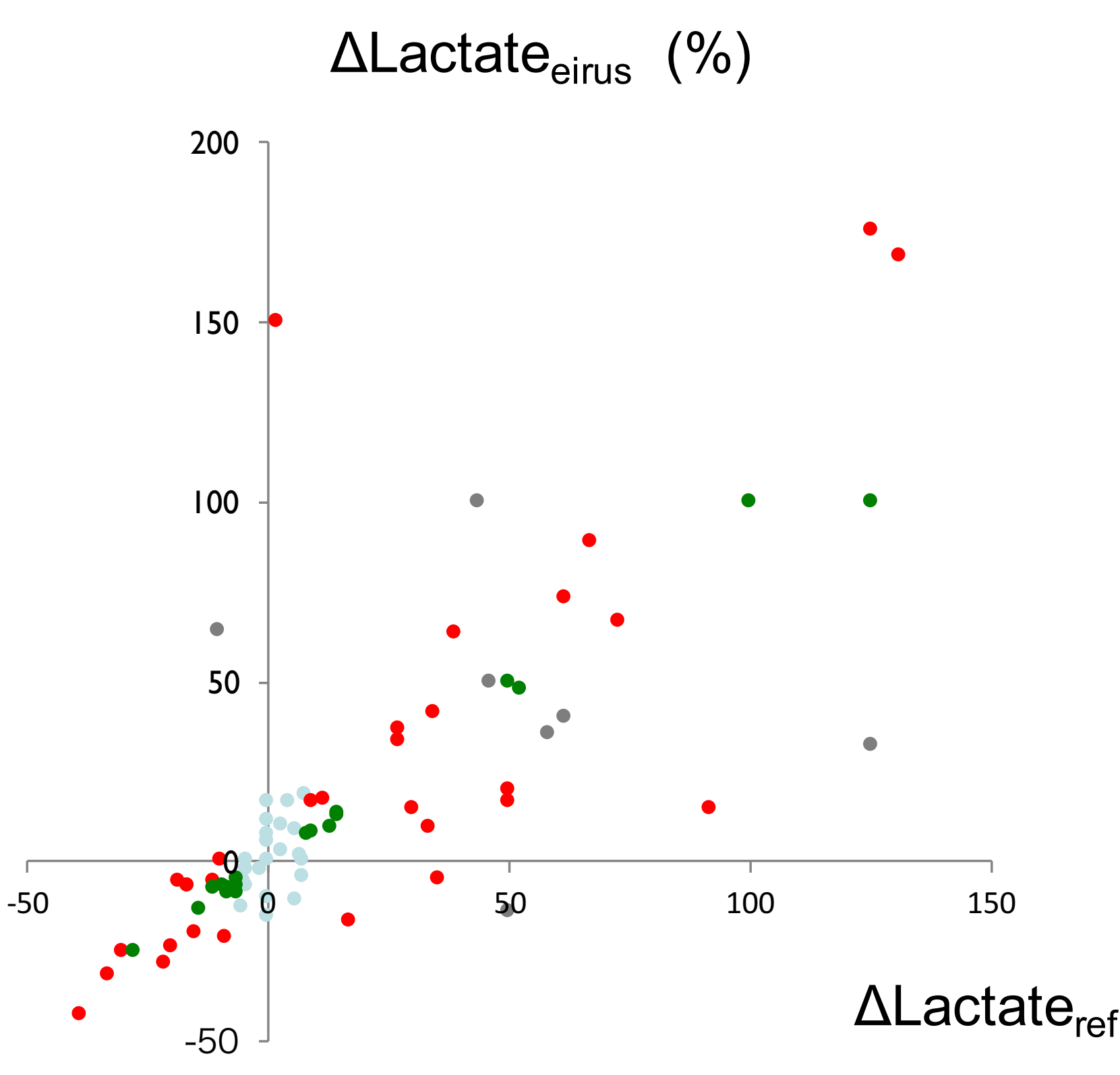

Conclusions: The microdialysis using a central venous catheter is safe, and seems reliable to absolute values of blood lactate. However, only one third of changes in lactate measurements are interchangeable with reference method.

Acknowledgments: Co authors list being restricted to 6 names, the following persons contributed equally to this study: Mrs Fradin Sabine, Dr Saplacan Vladimir, Pr Hanouz JeanLuc. 